\title{
Exactly solvable models of proton and neutron interacting bosons
}

\author{
S. Lerma H., ${ }^{1}$ B. Errea, ${ }^{1}$ J. Dukelsky, ${ }^{1}$ S. Pittel,,${ }^{1,2}$ and P. Van Isacker ${ }^{3}$ \\ ${ }^{1}$ Instituto de Estructura de la Materia, CSIC, Serrano 123, E-28006 Madrid, Spain \\ ${ }^{2}$ Bartol Research Institute and Department of Physics and Astronomy, University of Delaware, Newark, Delaware 19716, USA \\ ${ }^{3}$ Grand Accélérateur National d'Ions Lourds, BP 55027, F-14076 Caen Cedex 5, France
}

(Received 4 May 2006; published 22 August 2006)

\begin{abstract}
We describe a class of exactly-solvable models of interacting bosons based on the algebra $\operatorname{SO}(3,2)$. Each copy of the algebra represents a system of neutron and proton bosons in a given bosonic level interacting via a pairing interaction. The model that includes $s$ and $d$ bosons is a specific realization of the IBM2, restricted to the transition regime between vibrational and $\gamma$-soft nuclei. By including additional copies of the algebra, we can generate proton-neutron boson models involving other boson degrees of freedom, while still maintaining exact solvability. In each of these models, we can study not only the states of maximal symmetry, but also those of mixed symmetry, albeit still in the vibrational to $\gamma$-soft transition regime. Furthermore, in each of these models we can study some features of $F$-spin symmetry breaking. We report systematic calculations as a function of the pairing strength for models based on $s, d$, and $g$ bosons and on $s, d$, and $f$ bosons. The formalism of exactly-solvable models based on the $\mathrm{SO}(3,2)$ algebra is not limited to systems of proton and neutron bosons, however, but can also be applied to other scenarios that involve two species of interacting bosons.
\end{abstract}

DOI: 10.1103/PhysRevC.74.024314

PACS number(s): 21.60.Fw, 21.60.Ev, 03.65.Fd

\section{INTRODUCTION}

In the early 1960s, Richardson [1] showed how to exactly solve the pure pairing model including nondegenerate singleparticle orbits for both fermion [2] and boson systems [3]. In 2001, Dukelsky, Esebbag, and Schuck [4] showed how to generalize Richardson's solution, making use of analogous work by Gaudin [5] for spin models, so that the resulting Richardson-Gaudin models can now describe the physics of a wide variety of strongly-correlated many-body quantum systems governed by pairing correlations. Many interesting applications of these exactly-solvable models have been reported recently, to ultrasmall superconducting grains [6], to nuclear superconductivity [7], to the nuclear interacting boson model [8,9], and to Bose Einstein condensates [10]. The methods have been applied both to fermion and boson systems, invariably yielding useful insight into the properties of the complex quantum systems they model [11]. Furthermore, with slight generalization the methods have also proven useful in the description of mixed systems involving atoms (either bosonic or fermionic) coupled to molecular dimers in the presence of a Feshbach resonance [12].

All of the above models were based on the treatment of pairing correlations involving identical particles. The relevant algebraic structure for describing identical-particle pairing is $\mathrm{SU}(2)$ for fermions or SU(1,1) for bosons. Both are rank-one algebras with just one Cartan generator. There have been several efforts to derive exactly-solvable Richardson-Gaudin models based on larger rank algebras [13,14]. In particular, the rank-two $\mathrm{SO}(5)$ algebra describing isovector proton-neutron pairing was treated in Ref. [15]. More recently, the complete solution of the $\mathrm{SO}(5)$ Richardson-Gaudin model was presented in Ref. [16]. This made possible an exact treatment of isovector proton-neutron pairing in the presence of nondegenerate single-particle levels, which was used in a description of the $N=Z$ nucleus ${ }^{64} \mathrm{Ge}$ in an extended shell-model space.
In this paper, we describe the first example of an exactlysolvable Richardson-Gaudin model of interacting bosons that is based on a rank-two algebra. The model has the algebraic structure $\mathrm{SO}(3,2)$ and is of relevance to two-component boson systems. One possible realization is a mixture of ${ }^{97} \mathrm{Rb}$ atoms in the hyperfine states $\left|F=1, M_{f}=1\right\rangle,\left|F=1, M_{f}=-1\right\rangle$ [17]. While these systems have been studied [18] in mean-field approximation, more elaborate approximate methods or the use of exact solutions are needed to study effects beyond mean field. Here we will concentrate on a different realization, namely bosonic models of nuclei with distinct proton and neutron degrees of freedom. When only $s$ and $d$ bosons are included, the resulting models are restricted versions of the proton-neutron interacting boson model (IBM2) [19], in the transitional regime between $\gamma$-unstable and vibrational nuclei. At the same time, however, their generality makes it possible to address many issues that cannot be discussed within the context of the IBM2. In particular, the models can readily accommodate boson degrees of freedom other than just $s$ and $d$, still within a proton-neutron framework. As such, they can address issues related to the proton-neutron degree of freedom in boson models with $g$ bosons and/or $f$ bosons. The models can also accommodate $F$-spin breaking, which can thus likewise be studied in the presence of bosons other than $s$ and $d$.

The outline of the paper is as follows. We begin in Sec. II with a brief overview of the $\mathrm{SO}(3,2)$ algebra, confirming that it is indeed a rank-two algebra and obtaining its associated integrals of motion. In Sec. III, we show how this formalism can be used to build exactly-solvable models of relevance to interacting neutron and proton bosons in atomic nuclei. In Sec. IV we consider as specific examples models involving $s d, s d g$ and $s d f$ degrees of freedom and describe their exact solutions in a variety of scenarios. In Sec. V, we summarize the principal conclusions of this work. 


\section{SO(3,2) AND ITS CARTAN DECOMPOSITION}

In this section, we briefly review the elements of the Cartan decomposition of the $\operatorname{SO}(3,2)$ Lie algebra and show how it can be used to build the set of integrals of motion for models based on multiple copies of this algebra.

We will be considering a problem involving neutron and proton boson degrees of freedom, with creation and annihilation operators $l_{\rho m}^{\dagger}$ and $l_{\rho m}$, respectively. These operators create/annihilate a boson of angular momentum $l$, projection $m$ and type $\rho$.

Now consider the set of bilinear boson operators $\left(\rho, \rho^{\prime}=\right.$ $\nu$ or $\pi$ )

$$
\begin{aligned}
l_{\rho}^{\dagger} l_{\rho^{\prime}} & =\sum_{m} l_{\rho m}^{\dagger} l_{\rho^{\prime} m}, \\
b_{-1 l}^{\dagger} & =(-1)^{l / 2} l_{v}^{\dagger} \cdot l_{v}^{\dagger}, \\
b_{0 l}^{\dagger} & =(-1)^{l / 2} \sqrt{2} l_{\pi}^{\dagger} \cdot l_{v}^{\dagger}, \\
b_{+1 l}^{\dagger} & =(-1)^{l / 2} l_{\pi}^{\dagger} \cdot l_{\pi}^{\dagger},
\end{aligned}
$$

and the corresponding two-boson annihilation operators $b_{-1 l}, b_{0 l}, b_{+1 l}$. Here the scalar product $l_{\rho}^{\dagger} \cdot l_{\rho^{\prime}}^{\dagger}$ has the usual definition

$$
l_{\rho}^{\dagger} \cdot l_{\rho^{\prime}}^{\dagger}=(-1)^{l} \sum_{m}(-1)^{l-m} l_{\rho, m}^{\dagger} l_{\rho^{\prime},-m}^{\dagger} .
$$

For each $l$, there are four particle-hole operators, three pair creation operators, and three pair annihilation operators. All told, these ten operators generate an $\operatorname{SO}(3,2)$ algebra.

The Cartan decomposition of this algebra is achieved by rewriting the set of one-body operators as

$$
\begin{aligned}
& H_{l}^{1}=F_{z l}=\frac{1}{2}\left\{\sum_{m}\left(l_{\pi m}^{\dagger} l_{\pi m}-l_{v m}^{\dagger} l_{v m}\right)\right\}, \\
& H_{l}^{2}=\frac{1}{2}\left(l_{\pi}^{\dagger} l_{\pi}+l_{v}^{\dagger} l_{v}+(2 l+1)\right), \\
& F_{l}^{+}=l_{\pi}^{\dagger} l_{v}=(-1)^{l} l_{\pi}^{\dagger} \cdot \tilde{l}_{\nu}, \\
& F_{l}^{-}=l_{v}^{\dagger} l_{\pi}=(-1)^{l} l_{v}^{\dagger} \cdot \tilde{l}_{\pi} .
\end{aligned}
$$

It can be readily confirmed that $\left[H_{l}^{1}, H_{l}^{2}\right]=0$. As there are no other generators that simultaneously commute with both, $\mathrm{SO}(3,2)$ is a rank-two algebra and these two operators generate the Cartan subalgebra. (Note: the factor $1 / 2$ is included in the Cartan operators to ensure that the Cartan-Weyl basis is orthonormal with respect to the Killing form of Ref. [20].)

The remaining generators are the ladder operators of the algebra. $F_{l}^{+}$and $b_{l \mu}^{\dagger}$ are the raising operators and correspondingly $F_{l}^{-}$and $b_{l \mu}$ are the lowering operators.

Knowing the Cartan decomposition for each $l$, we can obtain the associated set of integrals of motion $R_{l}$, following Ref. [14]. The complete set of hermitean and mutually commuting operators with linear and quadratic terms in the rational model can be expressed in the form

$$
R_{l}=\frac{\Delta}{2 g} H_{l}^{1}+\frac{1}{2 g}(2+\Delta) H_{l}^{2}+\sum_{l^{\prime}(\neq l)} \frac{X_{l} \cdot X_{l^{\prime}}}{z_{l^{\prime}}-z_{l}}
$$

where $H_{l}^{a}(a=1,2)$ are the two Cartan operators of the copy $l$, and

$$
X_{l} \cdot X_{l^{\prime}}=F_{l} \cdot F_{l^{\prime}}+H_{l}^{2} H_{l^{\prime}}^{2}-\frac{1}{4} \sum_{\mu=-1,0,1}\left(b_{\mu l}^{\dagger} b_{\mu l^{\prime}}+b_{\mu l^{\prime}}^{\dagger} b_{\mu l}\right)
$$

For a problem involving $K$ copies of a rank-two $\mathrm{SO}(3,2)$ algebra the integrals of motion depend on a total of $K+2$ free parameters, the $K$ coefficients $z_{l}$ and the two parameters, $\Delta$ and $g$, that enter in the linear term of Eq. (3).

The exact solutions of the rational model correspond to solving the eigenvalue equations

$$
R_{l}\left|\Psi\left(e_{\alpha}, \omega_{\gamma}\right)\right\rangle=r_{l}\left|\Psi\left(e_{\alpha}, \omega_{\gamma}\right)\right\rangle .
$$

The eigenvalues $r_{l}$ can be written as

$$
\begin{aligned}
r_{l}= & \frac{1}{2 g}\left(U_{\pi l}+U_{\nu l}+\Delta U_{\pi l}\right)+\frac{1}{2 g}(2+\Delta) \Omega_{l} \\
& +\frac{1}{2} \sum_{l^{\prime}(\neq l)} \frac{1}{z_{l^{\prime}}-z_{l}}\left(\Omega_{l}\left(U_{\pi l^{\prime}}+U_{\nu l^{\prime}}\right)+\Omega_{l^{\prime}}\left(U_{\pi l}+U_{\nu l}\right)\right. \\
& \left.+2 \Omega_{l} \Omega_{l^{\prime}}+U_{\pi l} U_{\pi l^{\prime}}+U_{\nu l} U_{\nu l^{\prime}}\right)+\frac{1}{2}\left(U_{\pi l}-U_{\nu l}\right) \\
& \times \sum_{\gamma=1}^{M_{1}} \frac{1}{\omega_{\gamma}-z_{l}}+\left(U_{\nu l}+\Omega_{l}\right) \sum_{\alpha=1}^{M_{2}} \frac{1}{e_{\alpha}-z_{l}},
\end{aligned}
$$

where $U_{\rho l}(\rho=\pi$ and $\nu)$ are the number of unpaired $\rho$ bosons (i.e., the seniority) in the lowest weight state of the level $l$, and which define completely the $\operatorname{SO}(3,2)$ irreducible representation of copy $l$. Also, $\Omega_{l}=(2 l+1) / 2$ is proportional to the degeneracy of the levels, $M_{1}=N_{\pi}-\sum_{l} U_{\pi l}$ is the total number of paired $\pi$ bosons, and $M_{2} \equiv M=\frac{1}{2}\left(N_{\pi}-\right.$ $\left.\sum_{l} U_{\pi l}+N_{v}-\sum_{l} U_{v l}\right)$ is the total number of pairs. Finally, $\omega_{\gamma}\left(\gamma=1, \ldots, M_{1}\right)$, and $e_{\alpha}\left(\alpha=1, \ldots, M_{2}\right)$ are two sets of spectral parameters that must satisfy the set of coupled equations

$$
\begin{gathered}
\sum_{l} \frac{U_{\pi l}-U_{\nu l}}{z_{l}-\omega_{\gamma}}+\sum_{\delta(\neq \gamma)}^{M_{1}} \frac{2}{\omega_{\delta}-\omega_{\gamma}}-\sum_{\beta}^{M_{2}} \frac{2}{e_{\beta}-\omega_{\gamma}}=-\frac{\Delta}{g}, \\
\sum_{l} \frac{U_{v l}+\Omega_{l}}{z_{l}-e_{\alpha}}+\sum_{\beta(\neq \alpha)}^{M_{2}} \frac{2}{e_{\beta}-e_{\alpha}}-\sum_{\delta}^{M_{1}} \frac{1}{\omega_{\delta}-e_{\alpha}}=-\frac{1}{g} .
\end{gathered}
$$

These are generalized Richardson equations appropriate to the $\mathrm{SO}(3,2)$ algebra.

Once the free parameters that define the $R_{l}$ integrals of motion have been chosen, any linear combination,

$$
\sum_{l} \eta_{l} R_{l}
$$

of those integrals of motion also gives rise to an exactlysolvable model for the multiple copies of the $\mathrm{SO}(3,2)$ algebra, with eigenvalues obtained from the corresponding $r_{l}$ 's. Of course, any constant can be added to the Hamiltonian without affecting the exact solvability of the model.

For the scenario under discussion, a system of interacting neutron and proton bosons in several levels, it is obviously desirable that the exactly-solvable models that emerge have 
the appropriate single-particle energies for the various boson degrees of freedom. This is accomplished by requiring that the coefficients $\eta_{l}$ that define the linear combination of integrals of motion are proportional to the single-particle energies of the corresponding boson levels $\left(\eta_{l}=2 g \epsilon_{l}\right)$. If we furthermore choose the $z_{l}$ parameters that enter in the definition of the integrals of motion according to $z_{l}=2 \epsilon_{l}$, the two-body part of the model Hamiltonian likewise emerges in a very simple form, one in which the interaction matrix elements are level independent. Furthermore, the constant term that is added to the Hamiltonian can be chosen so as to leave it in a convenient form.

With these choices, the form of the Hamiltonian that emerges for multiple copies of the $\operatorname{SO}(3,2)$ algebra is

$$
\begin{aligned}
H= & 2 g \sum_{l} \epsilon_{l} R_{l}+c \hat{1} \\
= & \sum_{l} \epsilon_{l}\left(\hat{N}_{\pi l}+\hat{N}_{\nu l}+\Delta \hat{N}_{\pi l}\right)+\frac{g}{4} \sum_{l l^{\prime} \mu} b_{\mu l}^{\dagger} b_{\mu l^{\prime}} \\
& -\frac{g}{2} F \cdot F+\frac{g}{2}\left(B\left(U_{\pi}, U_{\nu}\right)+C\right) \hat{1},
\end{aligned}
$$

where

$$
F=\sum_{l} F_{l},
$$

$c=g \sum_{l^{\prime}>l} \Omega_{l} \Omega_{l^{\prime}}-(2+\Delta) \sum_{l} \epsilon_{l} \Omega_{l}$ is a constant, and $\hat{1}$ is the identity operator.

The diagonal term in the last line of Eq. (9) depends on

$B\left(U_{\pi}, U_{v}\right)=\sum_{l}\left(\frac{U_{\pi l}^{2}+U_{v l}^{2}}{2}+\left(U_{\pi l}+U_{v l}\right) \Omega_{l}-U_{v l}-2 U_{\pi l}\right)$,

a function of the seniorities, and a constant $C=N(4 \Omega+6-$ $N) / 4$, with $\Omega \equiv \sum_{l} \Omega_{l}$, and $N$ the total number of bosons $\left(N \equiv \sum_{l} N_{\pi l}+\sum_{l} N_{\nu l}\right)$.

The eigenvalues of the above Hamiltonian $\left(2 g \sum_{l} \epsilon_{l} r_{l}+c\right)$ can be expressed as

$$
\begin{aligned}
E= & \sum_{l} \epsilon_{l}\left(U_{\pi l}+U_{\nu l}+\Delta U_{\pi l}\right)+\sum_{\alpha} e_{\alpha}+\frac{g}{2}\left[\frac{\Delta}{g} \sum_{\gamma} \omega_{\gamma}\right. \\
& \left.-\left(-F_{z}\right)\left(-F_{z}+1\right)\right]+\frac{g}{2}\left(B\left(U_{\pi}, U_{\nu}\right)+C\right),
\end{aligned}
$$

where $F_{z}=\left(N_{\pi}-N_{\nu}\right) / 2$. The first term on the right hand side is the single particle energy of the unpaired bosons, the second one is the energy of the boson pairs, whereas the term in brackets is related to $F$-spin symmetry. It is possible to show that in the $F$-spin-symmetric limit ( $\Delta \rightarrow 0$ ), both terms in the brackets combine to give

$$
\lim _{\Delta \rightarrow 0}\left[\frac{\Delta}{g} \sum_{\gamma} \omega_{\gamma}-\left(-F_{z}\right)\left(-F_{z}+1\right)\right]=-F(F+1) .
$$

It is especially convenient to rewrite the Hamiltonian (9) in multipole-multipole form, to facilitate a link to the traditional phenomenological Hamiltonians used in bosonic descriptions of nuclei. The Hamiltonian (9) expressed in terms of multipoles can be written as

$$
\begin{aligned}
H= & \sum_{l} \epsilon_{l}\left(\hat{N}_{\pi l}+\hat{N}_{\nu l}+\Delta \hat{N}_{\pi l}\right)-\frac{g}{4} \sum_{l<l^{\prime}} \sum_{L=\left|l^{\prime}-l\right|}^{l^{\prime}+l}(-)^{L} \\
& \times\left(Q_{l_{v} l_{v}^{\prime}}^{L}+Q_{l_{\pi} l_{\pi}^{\prime}}^{L}\right) \cdot\left(Q_{l_{v}^{\prime} l_{v}}^{L}+Q_{l_{\pi}^{\prime} l_{\pi}}^{L}\right)
\end{aligned}
$$

where

$$
Q_{l_{\rho} l_{\rho}^{\prime}}^{L}=\left(l_{\rho}^{\dagger} \tilde{l}_{\rho}^{\prime}-(-)^{l+\frac{l+l^{\prime}}{2}} l_{\rho}^{\prime \dagger} \tilde{l}_{\rho}\right)^{L} .
$$

\section{SO(3,2) AND THE PROTON-NEUTRON INTERACTING BOSON MODEL}

The multicopy $\mathrm{SO}(3,2)$ formalism described in the preceding section has relevance to the proton-neutron interacting boson model of atomic nuclei [19]. In this model, distinct neutron and proton bosons are introduced to model collective pairs of identical nucleons. The standard version of the model is limited to $s$ and $d$ bosons, reflecting the fact that the energetically lowest pairs of identical nucleons are invariably those with $J^{P}=0^{+}$and $J^{P}=2^{+}$and that they are typically well separated from all higher pairs. The resulting model, usually called the IBM2, has been used to describe successfully the collective properties of nuclei throughout the periodic table.

Soon after the introduction of the IBM2, it became clear that there was a natural connection of the model both to the nuclear shell model and to the simpler IBM1 model with but one kind of boson.

The relationship to the shell model is contained in the association of the bosons with the lowest collective pairs of identical nucleons. Thus, it is possible to use input from the shell model to help define the Hamiltonian that acts in the IBM2 model space.

The relationship to the IBM1 comes through the introduction of a quantum number called $F$ spin that distinguishes the two species in IBM2, the neutron and proton bosons. In an IBM2 Hamiltonian that conserves this quantum number, the lowest states of the system are those that are maximally symmetric in $F$-spin, in other words maximally symmetric under the interchange of neutron and proton bosons. It is these states that are modelled by the simpler IBM1. However, the IBM2 also contains states that are not maximally symmetric under the interchange of neutron and proton bosons, states that are said to have mixed symmetry. In an $F$-spin conserving version of IBM2, these states are decoupled from the states of maximal $F$-spin symmetry.

With the above as background, we are now in a position to discuss the relationship between the exactly-solvable models that derive from the $\mathrm{SO}(3,2)$ algebra and the IBM2. In particular, if we limit our discussion to two copies of the algebra, one involving an $l=0 \mathrm{~s}$ boson and the other involving an $l=2 d$ boson, then we arrive precisely at a Hamiltonian of the IBM2 form. It is, however, not the most general IBM2 Hamiltonian. Rather, as we will see shortly it is a Hamiltonian that models the transition between vibrational $(\mathrm{U}(5))$ and $\gamma$-soft $(\mathrm{SO}(6))$ nuclei within an IBM2 framework. 
The Hamiltonian that derives in a two-copy $s-d$ realization of $\operatorname{SO}(3,2)$ has the form [see Eqs. (13) and (14), with $l, l^{\prime}=$ $s, d$ only, and taking $\left.\epsilon_{s}=0\right]$

$$
\begin{aligned}
H= & \epsilon_{d}\left(\hat{N}_{\pi d}+\hat{N}_{\nu d}+\Delta \hat{N}_{\pi d}\right) \\
& -\frac{g}{4}\left(\hat{Q}_{\pi}^{2}+\hat{Q}_{v}^{2}\right) \cdot\left(\hat{Q}_{\pi}^{2}+\hat{Q}_{v}^{2}\right),
\end{aligned}
$$

where

$$
\hat{Q}_{\rho}^{2}=s_{\rho}^{\dagger} \tilde{d}_{\rho}+d_{\rho}^{\dagger} \tilde{s}_{\rho} .
$$

Its eigenvalues can be obtained from Eq. (12).

There are a couple of features of this Hamiltonian worthy of note. The first is that as mentioned above it is not a completely general IBM2 Hamiltonian, as the quadrupole operator is restricted to $\chi=0$. Second, the Hamiltonian contains a term $\Delta \hat{N}_{\pi d}$, which arises if the single-boson splittings between the $s$ and $d$ levels in the two species (neutrons and protons) are different, and which will in general break $F$-spin symmetry. $F$-spin symmetry is only preserved if $\Delta=0$. Finally, the quadrupole-quadrupole interaction that enters is an $F=0$ tensor, with a definite relation between the interactions among the different species. There is no additional Majorana interaction in the model, as the term $g F \cdot F$ in Eq. (9) is absorbed completely into the $F$-spin-scalar quadrupole-quadrupole interaction. However, when $\Delta=0$, i.e., when $F$-spin symmetry is preserved, we can add an additional Majorana term of arbitrary strength while maintaining the exact solvability of the model.

Perhaps the key feature of the exactly-solvable protonneutron boson models that derive from the multi-copy $\operatorname{SO}(3,2)$ algebra is that they can accommodate boson degrees of freedom other than just the $s$ and $d$. Indeed, they can accommodate any number of boson degrees of freedom, albeit with the restricted Hamiltonians that can be accessed. As an example, the model with $s, d$ and $g$ degrees of freedom is of the form $\left(\epsilon_{s}=0\right)$

$$
\begin{aligned}
H= & \sum_{l=d, g} \epsilon_{l}\left(\hat{N}_{\pi l}+\hat{N}_{\nu l}+\Delta \hat{N}_{\pi l}\right) \\
& -\frac{g}{4} \sum_{l<l^{\prime}} \sum_{L=2, \ldots, 6}(-)^{L}\left(Q_{l_{v} l_{v}^{\prime}}^{L}+Q_{l_{\pi} l_{\pi}^{\prime}}^{L}\right) \cdot\left(Q_{l_{v}^{\prime} l_{v}}^{L}+Q_{l_{\pi}^{\prime} l_{\pi}}^{L}\right) .
\end{aligned}
$$

Note that it contains all multipole interactions from $L=2$ to $L=6$. Once again, all enter as $F=0$ tensors, with no additional Majorana interaction present. Here too, however, a Majorana interaction of arbitrary strength can be included if $\Delta=0$ so that $F$-spin is conserved.

Likewise, we can accommodate $f$ bosons, either with or without $g$ bosons, still in a proton-neutron boson framework. When considering $s d$ and $f$ bosons, for example, the class of Hamiltonians that arise are $\left(\epsilon_{s}=0\right)$

$$
\begin{aligned}
H= & \sum_{l=d, f} \epsilon_{l}\left(\hat{N}_{\pi l}+\hat{N}_{v l}+\Delta \hat{N}_{\pi l}\right)-\frac{g}{4} \sum_{l<l^{\prime}} \sum_{L=1, \ldots, 5}(-)^{L} \\
& \times\left(Q_{l_{v} l_{v}^{\prime}}^{L}+Q_{l_{\pi} l_{\pi}^{\prime}}^{L}\right) \cdot\left(Q_{l_{v}^{\prime} l_{v}}^{L}+Q_{l_{\pi}^{\prime} l_{\pi}}^{L}\right) .
\end{aligned}
$$

Note that now we only have multipoles from $L=1$ through 5. The $L=1,3,4$, and 5 multipoles all have odd parity; the
$L=2$ multipole has an even-parity component $\left(l, l^{\prime}=s, d\right)$ and an odd-parity component $\left(l, l^{\prime}=d, f\right)$.

\section{APPLICATIONS OF EXACTLY-SOLVABLE PROTON-NEUTRON INTERACTING BOSON MODELS DERIVED FROM THE SO $(3,2)$ ALGEBRA}

In this section, we apply the various exactly-solvable model Hamiltonians developed in the previous section to scenarios of relevance to the collective structure of atomic nuclei.

We first consider a model of $s, d$ and $g$ interacting neutron and proton bosons, for which the relevant Hamiltonian was given in Eq. (17). We compare the results for this model with those of the corresponding model with just $s$ and $d$ bosons, [see Eq. (15)]. The analysis is carried out as a function of the pairing strength $g$, for fixed single-boson energies, with the results shown in Fig. 1.

The complete Hilbert space of the model can be split into invariant subspaces characterized by the seniority numbers $\left(U_{\pi l}, U_{v l}\right)$. For each seniority, one has to solve a different set of Richardson equations (7) to obtain the eigenvalues (12). The angular momenta, parities, and $F$-spin quantum numbers associated with the set of seniorities we consider are shown in Table I.

The top panel in Fig. 1 gives the results for the $s d$ model and the bottom panel gives the $s d g$ results. All energies are measured with respect to that of the ground state.

In the case of the $s d$ model, we use $\epsilon_{d}=1 \mathrm{MeV}$ and $\epsilon_{s}=$ $0 \mathrm{MeV}$, and the relevant seniorities are those with $U_{g}=0$ in Table I. For the $s d g$ model, we again use $\epsilon_{d}=1 \mathrm{MeV}$, and $\epsilon_{s}=0 \mathrm{MeV}$, but now include a $g$ boson at $\epsilon_{g}=1.6 \mathrm{MeV}$. In this case, we consider all the seniorities of Table I. The level energies chosen are physically reasonable for the various boson degrees of freedom. In both calculations, we consider a system of $N=20$ bosons, with $N_{v}=N_{\pi}=10\left(F_{\max }=10\right)$. Furthermore, we assume $\Delta=0$ so that $F$-spin symmetry is

\begin{tabular}{|c|c|c|}
\hline$\left(U_{\pi s} U_{\nu s} U_{\pi d} U_{\nu d} U_{\pi g} U_{\nu g}\right)$ & $F$ & $J^{P}$ \\
\hline$\left(\begin{array}{llllll}0 & 0 & 0 & 0 & 0 & 0\end{array}\right)$ & $0,1, \ldots, F_{\max }$ & $0^{+}$ \\
\hline$\left(\begin{array}{llllll}0 & 1 & 0 & 1 & 0 & 0\end{array}\right)$ & $0,1, \ldots, F_{\max }$ & $2^{+}$ \\
\hline$\left(\begin{array}{llllll}0 & 1 & 0 & 0 & 0 & 1\end{array}\right)$ & $0,1, \ldots, F_{\max }$ & $4^{+}$ \\
\hline$\left(\begin{array}{llllll}0 & 0 & 0 & 2 & 0 & 0\end{array}\right)$ & $0,1, \ldots, F_{\max }$ & $2^{+}, 4^{+}$ \\
\hline$\left(\begin{array}{llllll}0 & 0 & 1 & 1 & 0 & 0\end{array}\right)$ & $0,1, \ldots, F_{\max }-1$ & $1^{+}, 3^{+}$ \\
\hline$\left(\begin{array}{llllll}0 & 0 & 0 & 1 & 0 & 1\end{array}\right)$ & $0,1, \ldots, F_{\max }$ & $2^{+}, 3^{+}, 4^{+}, 5^{+}, 6^{+}$ \\
\hline$\left(\begin{array}{llllll}0 & 0 & 0 & 0 & 0 & 2\end{array}\right)$ & $0,1, \ldots, F_{\max }$ & $2^{+}, 4^{+}, 6^{+}, 8^{+}$ \\
\hline$\left(\begin{array}{llllll}0 & 0 & 0 & 0 & 1 & 1\end{array}\right)$ & $0,1, \ldots, F_{\max }-1$ & $1^{+}, 3^{+}, 5^{+}, 7^{+}$ \\
\hline$\left(\begin{array}{llllll}0 & 1 & 0 & 3 & 0 & 0\end{array}\right)$ & $0,1, \ldots, F_{\max }$ & $0^{+}, 3^{+}, 4^{+}, 6^{+}$ \\
\hline$\left(\begin{array}{llllll}0 & 0 & 0 & 4 & 0 & 0\end{array}\right)$ & $0,1, \ldots, F_{\max }$ & $2^{+}, 4^{+}, 5^{+}, 6^{+}, 8^{+}$ \\
\hline$\left(\begin{array}{llllll}0 & 1 & 0 & 2 & 0 & 1\end{array}\right)$ & $0,1, \ldots, F_{\max }$ & $0^{+}, 1^{+}, \ldots, 8^{+}$ \\
\hline$\left(\begin{array}{llllll}0 & 1 & 0 & 1 & 0 & 2\end{array}\right)$ & $0,1, \ldots, F_{\max }$ & $0^{+}, 1^{+}, \ldots, 10^{+}$ \\
\hline
\end{tabular}
preserved.

Note that $g=0$ corresponds to the precise U(5) limit in the $s d$ model and to the corresponding vibrational limit in

TABLE I. Seniorities considered in the $s d$ and $s d g$ calculations and their associated angular momenta, parities and $F$-spin quantum numbers. 

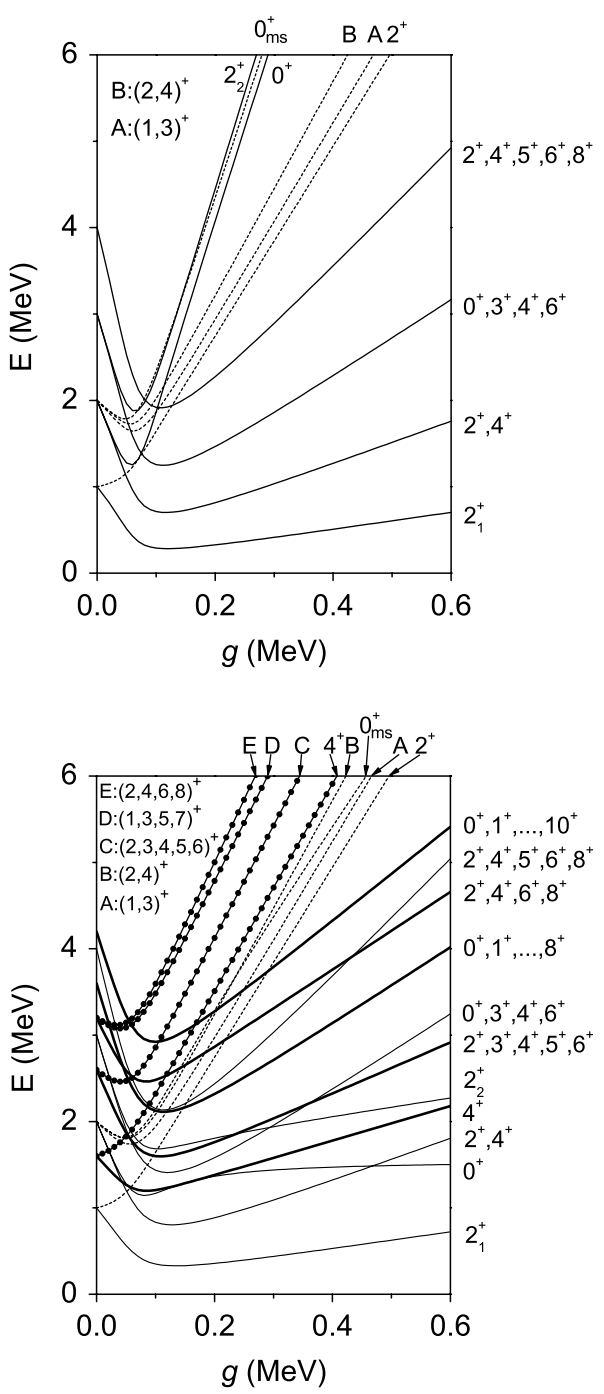

FIG. 1. Excitation energies of the $s d g$ Hamiltonian in Eq. (17) (bottom panel) compared to those of the $s d$ Hamiltonian in Eq. (15) (top panel), as a function of the interaction strength $g$ and for the seniorities of Table I. Continuous lines correspond to maximal $F$-spin states, dashed and circled lines refer to mixed symmetry states $(F=$ $\left.F_{\max }-1\right)$. Thick and circled lines in the bottom panel indicate states with unpaired $g$ bosons $\left(U_{g} \neq 0\right)$, for maximal and mixed symmetry states respectively. Except for $J^{P}=2^{+}$, only the first excited state of each seniority is shown. Only those mixed symmetry states up to total seniority two are presented. The single-boson energies are given in the text.

the $s d g$ model. As the pairing strength $g$ increases, there is a phase transition to a $\gamma$-unstable system, occurring at roughly $g=0.08 \mathrm{MeV}$.

Some interesting features can be noted in these results. Inclusion of the $g$ level in the calculation increases significantly the number of possible $J^{P}$ states (indicated by thick and circled lines in the bottom panel of Fig. 1, see Table I), but otherwise leaves most of the states of the $s d$ model relatively unaffected. For a few states, however, and in particular the levels denoted $0^{+}, 0_{\mathrm{ms}}^{+}$, and $2_{2}^{+}$, there are important and interesting changes that take place. As an example, consider the first excited $0^{+}$ state. In the $s d$ calculation, its energy goes up as $g$ increases;
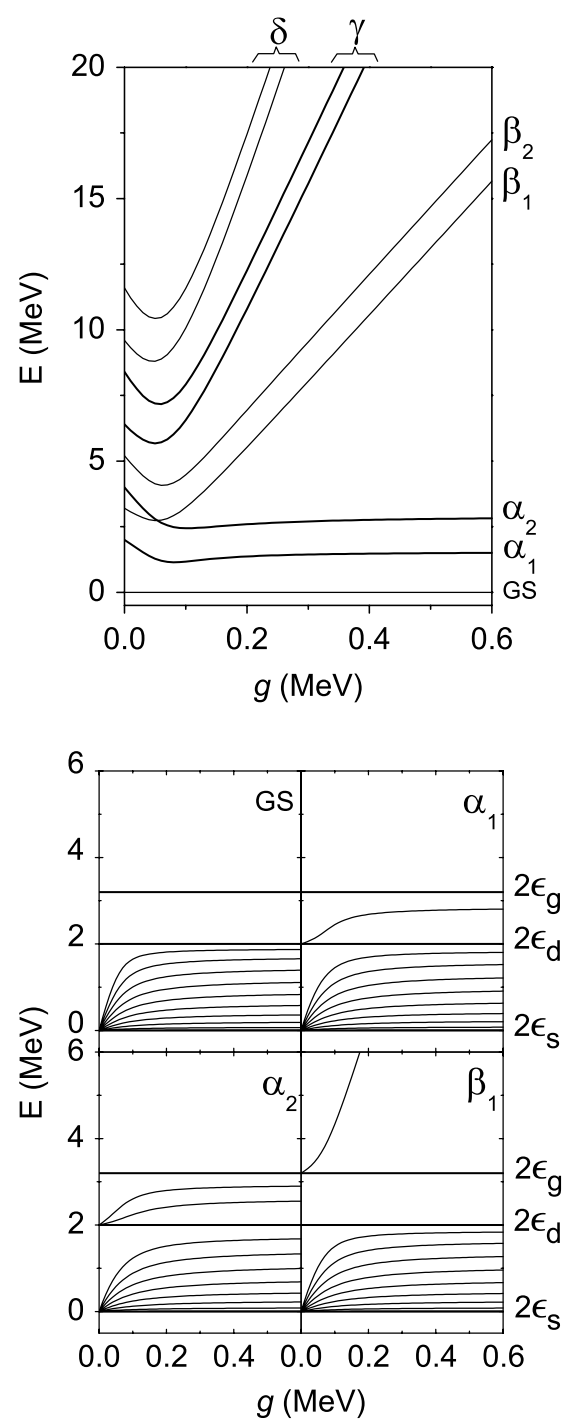

FIG. 2. Excitation energies of the $s d g$ Hamiltonian in Eq. (17) (top panel) for seniority-zero states $\left(J^{P}=0^{+}\right)$. The thickness of the lines serves as a guide to the eye to distinguish the band structure of the spectrum $(\alpha, \beta, \gamma, \delta)$. Only the first two states for each band are plotted. In the bottom panel the pair energies $\left(e_{\alpha}, \alpha=1, \ldots, 10\right)$ associated with the ground state (GS), the first $\left(\alpha_{1}\right)$ and second $\left(\alpha_{2}\right)$ states of the lowest excited band, and the first $\left(\beta_{1}\right)$ state of the second excited band are shown (see text for details). All the parameters are the same as for the bottom panel of Fig. 1.

in the corresponding $s d g$ calculation its energy flattens out. A similar effect can be seen for the $0_{\mathrm{ms}}^{+}$and $2_{2}^{+}$states, which likewise are flattened out in energy.

To better illustrate this phenomenon and how it arises, we present in the bottom panel of Fig. 2 the pair energies $\left(e_{\alpha}\right)$ associated with some selected seniority-zero states $\left(J^{P}=0^{+}\right)$ of the $s d g$ model, as a function of the interaction strength. We also show the associated spectrum of levels in the top panel of the figure.

For the ground state of the system (GS), all pair energies are trapped between $2 \epsilon_{d}$ and $2 \epsilon_{s}$. Then there are a series of levels in which one or more of the pair energies are promoted into the energy region from $2 \epsilon_{d}$ to $2 \epsilon_{g}$. The corresponding 
levels are likewise trapped, as their energies are simply a sum of the associated pair energies. The lowest is $\alpha_{1}$, with but one pair excited. The next is $\alpha_{2}$, with two pairs excited. And as should be evident there are many other such states, in which at least one pair is between $2 \epsilon_{g}$ and $2 \epsilon_{d}$, but in which none are above $2 \epsilon_{g}$. For simplicity, however, we only show the lowest two levels of this band.

Next we consider levels in which one pair is excited above $2 \epsilon_{g}$. The lowest such level is denoted $\beta_{1}$ in both panels. Since the last pair is not trapped, this level goes up in energy as $g$ increases. Furthermore, there will be a band of levels in which just one pair has been excited above $2 \epsilon_{g}$. Once again, however, we only show the two lowest states of the band.

Finally, we show two other bands in the spectrum, denoted $\gamma$ and $\delta$. They correspond to two and three pairs above $2 \epsilon_{g}$, respectively. They too are unbounded as a function of $g$. Of course there are higher bands as well, corresponding to progressively more pairs being excited above $2 \epsilon_{g}$.

As should be clear from the figures and the discussion, this unusual behavior for selected levels occurs whenever there are more than two boson levels active and a repulsive boson-boson pairing interaction [9].

As noted earlier, the trapping of pair energies is not restricted to $0^{+}$states. Indeed, the band structure found for seniority-zero states is a feature common to all seniority sectors. However, for the states with seniority different from zero the term $(g / 2) B\left(U_{\pi}, U_{v}\right)$ in the eigenvalues (12) also contributes, making the excitation energies for such states unbounded as a function of the interaction strength $g$. Nevertheless, these states likewise arise from trapped pair energies and thus are also flattened out as a function of $g$.

For the mixed-symmetry states in the $\Delta=0$ limit, the term in brackets in Eq. (12) produces a contribution to the excitation energy,

$$
\frac{g}{2}\left(F_{\max }\left(F_{\max }+1\right)-F(F+1)\right),
$$

thereby explaining why they appear at higher energy than the maximal $F$-spin states. As was mentioned above, however, when $\Delta=0$ it is possible to add a Majorana term of arbitrary strength, and thus move the location of the mixed-symmetry states at will with respect to those of maximal $F$-spin.

We next consider the effect of including an $f$ boson, focussing now on an $s d f$ boson model. In Fig. 3, we present the results for this model as a function of $g$, for the case of $\epsilon_{s}=0 \mathrm{MeV}, \epsilon_{d}=1 \mathrm{MeV}$ and $\epsilon_{f}=1.6 \mathrm{MeV}$. The seniorities that were considered in this calculation are listed in Table II.

Thus, this model is very similar to the $s d g$ model shown in Fig. 1, with the $f$ boson replacing the $g$ boson at precisely the same energy. Here too the second $0^{+}$level is trapped, as this is a general phenomenon that occurs whenever there are more than two boson degrees of freedom and a repulsive pairing interaction. Indeed, the spectra for the $s d f$ and $s d g$ models as a function of $g$ are very similar. The exactly-solvable $\mathrm{SO}(3,2)$ Richardson-Gaudin models do not couple states with broken pairs, which is where differences would have shown up.

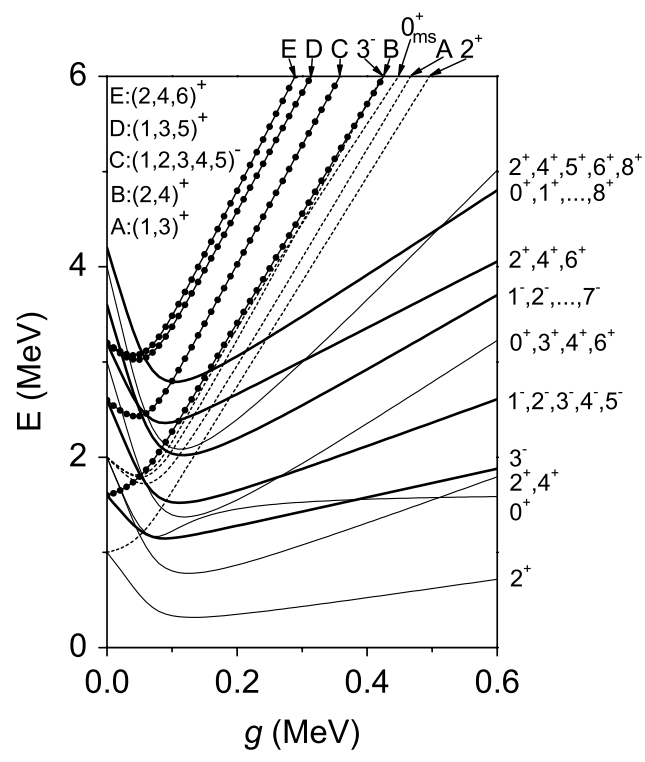

FIG. 3. Excitation energies of the $s d f$ Hamiltonian in Eq. (18) as a function of the interaction strength $g$, for the seniorities of Table II. Continuous lines correspond to maximal $F$-spin states, dashed and circled lines refer to mixed symmetry states $\left(F=F_{\max }-1\right)$. Thick and circled lines indicate states with unpaired $f$ bosons $\left(U_{f} \neq 0\right)$, for maximal and mixed $F$-symmetry states, respectively. Only the first excited sate of each seniority is shown. For mixed symmetry states, only states up to total seniority two are plotted. The single-boson energies are given in the text.

\section{SUMMARY AND CONCLUSIONS}

In this paper, we have described the first example of an exactly-solvable Richardson-Gaudin model based on a rank-two algebra for bosons. The example we discussed involved multiple copies of the $\mathrm{SO}(3,2)$ algebra. We focused on a specific realization involving proton and neutron bosons subject to a pairing interaction. The models that emerged are exactly solvable for nondegenerate single-boson energies and for any number of bosonic copies. When only $s$ and $d$ bosons are included, the resulting model is a specific example of the proton-neutron interacting boson model (IBM2), limited

TABLE II. Seniorities considered in the $s d f$ calculations and their associated angular momenta, parities and $F$-spin quantum numbers.

\begin{tabular}{|c|c|c|}
\hline$\left(U_{\pi s} U_{\nu s} U_{\pi d} U_{v d} U_{\pi f} U_{v f}\right)$ & $F$ & $J^{P}$ \\
\hline$\left(\begin{array}{llllll}0 & 0 & 0 & 0 & 0 & 0\end{array}\right)$ & $0,1, \ldots, F_{\max }$ & $0^{+}$ \\
\hline$\left(\begin{array}{llllll}0 & 1 & 0 & 1 & 0 & 0\end{array}\right)$ & $0,1, \ldots, F_{\max }$ & $2^{+}$ \\
\hline$\left(\begin{array}{llllll}0 & 1 & 0 & 0 & 0 & 1\end{array}\right)$ & $0,1, \ldots, F_{\max }$ & $3^{-}$ \\
\hline$\left(\begin{array}{llllll}0 & 0 & 0 & 2 & 0 & 0\end{array}\right)$ & $0,1, \ldots, F_{\max }$ & $2^{+}, 4^{+}$ \\
\hline$\left(\begin{array}{llllll}0 & 0 & 1 & 1 & 0 & 0\end{array}\right)$ & $0,1, \ldots, F_{\max }-1$ & $1^{+}, 3^{+}$ \\
\hline$\left(\begin{array}{llllll}0 & 0 & 0 & 1 & 0 & 1\end{array}\right)$ & $0,1, \ldots, F_{\max }$ & $1^{-}, 2^{-}, 3^{-}, 4^{-}, 5^{-}$ \\
\hline$\left(\begin{array}{llllll}0 & 0 & 0 & 0 & 0 & 2\end{array}\right)$ & $0,1, \ldots, F_{\max }$ & $2^{+}, 4^{+}, 6^{+}$ \\
\hline$\left(\begin{array}{llllll}0 & 0 & 0 & 0 & 1 & 1\end{array}\right)$ & $0,1, \ldots, F_{\max }-1$ & $1^{+}, 3^{+}, 5^{+}$ \\
\hline$\left(\begin{array}{llllll}0 & 1 & 0 & 3 & 0 & 0\end{array}\right)$ & $0,1, \ldots, F_{\max }$ & $0^{+}, 3^{+}, 4^{+}, 6^{+}$ \\
\hline$\left(\begin{array}{llllll}0 & 0 & 0 & 4 & 0 & 0\end{array}\right)$ & $0,1, \ldots, F_{\max }$ & $2^{+}, 4^{+}, 5^{+}, 6^{+}, 8^{+}$ \\
\hline$\left(\begin{array}{llllll}0 & 1 & 0 & 2 & 0 & 1\end{array}\right)$ & $0,1, \ldots, F_{\max }$ & $1^{-}, 2^{-}, \ldots, 7^{-}$ \\
\hline$\left(\begin{array}{llllll}0 & 1 & 0 & 1 & 0 & 2\end{array}\right)$ & $0,1, \ldots, F_{\max }$ & $0^{+}, 1^{+}, \ldots, 8^{+}$ \\
\hline
\end{tabular}


however to the transition from vibrational to $\gamma$-soft motion. Other bosonic degrees of freedom, such as the $g$ and/or $f$, can be readily included while still preserving the exact solvability of the model. Through the use of these models, we can address issues related to the role of mixed-symmetry states in proton-neutron boson models with many boson degrees of freedom. We can even study some limited features associated with $F$-spin symmetry breaking for these multicopy boson models.

One issue addressed here concerned the role of the $g$ boson in proton-neutron boson models. We compared the results of $s d$ calculations with those of $s d g$ calculations for an $F$-spin-symmetric Hamiltonian, throughout the vibrational to $\gamma$-soft transition region, and saw that in the presence of a $g$ boson several low-lying excited states have their properties dramatically modified as the result of a trapping phenomenon for the pair energies.

We also studied the role of the $f$ boson, in the context of an $s d f$ model of interacting proton and neutron bosons. Because of some special features of the exactly-solvable models that we treat, there were no meaningful differences between the $s d g$ and $s d f$ results, except of course as regards the angular momentum content of the collective states.
The models that we have developed are not limited, however, to proton-neutron boson models of nuclei. Any physical problem involving two species of bosons in which pairing is dominant can be modelled in this way. In Sec. I, we noted the possibility of applying these models to problems involving a mixture of ${ }^{97} \mathrm{Rb}$ atoms in the hyperfine states $\left|F=1, M_{f}=1\right\rangle,\left|F=1, M_{f}=-1\right\rangle$. The availability of an exactly-solvable model with which to study such systems, makes it possible to study effects that go beyond the limited mean-field descriptions considered to date.

\section{ACKNOWLEDGMENTS}

This work was supported in part by the Spanish DGI under grant No. BFM2003-05316-C02-02, by the U.S. National Science Foundation under grant Nos. PHY-0140036 and PHY-0553127 and by a CICYT-IN2P3 cooperation. S.L.H. has a post-doctoral grant from the Spanish SEUI-MEC and B.E. has a predoctoral grant from the Spanish CE-CAM. S.P. would like to acknowledge the hospitality and support of the CSIC, Madrid, where most of this work was carried out.
[1] R. W. Richardson, Phys. Lett. 3, 277 (1963).

[2] R. W. Richardson, Phys. Rev. 141, 949 (1966).

[3] R. W. Richardson, J. Math. Phys. 9, 1327 (1968).

[4] J. Dukelsky, C. Esebbag, and P. Schuck, Phys. Rev. Lett. 87, 066403 (2001).

[5] M. Gaudin, J. Phys. (Paris) 37, 1087 (1976).

[6] G. Sierra, J. Dukelsky, G. G. Dussel, J. von Delft, and F. Braun, Phys. Rev. B 61, R11890 (2000).

[7] J. Dukelsky, C. Esebbag, and S. Pittel, Phys. Rev. Lett. 88, 062501 (2002).

[8] F. Pan and J. P. Draayer, Nucl. Phys. A636, 156 (1998).

[9] J. Dukelsky and S. Pittel, Phys. Rev. Lett. 86, 4791 (2001).

[10] J. Dukelsky and P. Schuck, Phys. Rev. Lett. 86, 4207 (2001).

[11] J. Dukelsky, S. Pittel, and G. Sierra, Rev. Mod. Phys. 76, 643 (2004); G. Ortiz, R. Somma, J. Dukelsky, and S. Rombouts, Nucl. Phys. B707, 421 (2005).

[12] J. Dukelsky, G. G. Dussel, C. Esebbag, and S. Pittel, Phys. Rev. Lett. 93, 050403 (2004).
[13] A. G. Usveridze, Quasi-Exactly Solvable Models in Quantum Mechanics (Institute of Physics Publishing, Bristol, 1994).

[14] M. Asorey, F. Falceto, and G. Sierra, Nucl. Phys. B622, 593 (2002).

[15] R. W. Richardson, Phys. Rev. 144, 874 (1966); F. Pan and J. P. Draayer, Phys. Rev. C 66, 044314 (2002); J. Links, H.-Q. Zhou, M. D. Gould, and R. H. McKenzie, J. Phys. A 35, 6459 (2002).

[16] J. Dukelsky, V. G. Gueorguiev, P. Van Isacker, S. Dimitrova, B. Errea, and S. Lerma H., Phys. Rev. Lett. 96, 072503 (2006).

[17] C. J. Myatt, E. A. Burt, R. W. Ghrist, E. A. Cornell, and C. E. Wieman, Phys. Rev. Lett. 78, 586 (1997).

[18] S. Ashhab and A. J. Leggett, Phys. Rev. A 68, 063612 (2003); Yu Shi and Q. Niu, Phys. Rev. Lett. 96, 140401 (2006).

[19] F. Iachello and A. Arima, The Interacting Boson Model (Cambridge University Press, Cambridge, 1987).

[20] P. Di Francesco, P. Mathieu, and D. Sénéchal, Conformal Field Theory (Springer, New York, 1997). 